\title{
Does an additional structured information program during the intensive care unit stay reduce anxiety in ICU patients?: a multicenter randomized controlled trial
}

Steffen Fleischer ${ }^{1 *}$, Almuth Berg ${ }^{1}$, Johann Behrens ${ }^{1}$, Oliver Kuss², Ralf Becker ${ }^{3}$, Annegret Horbach ${ }^{4,5,6}$ and Thomas R Neubert ${ }^{7,8}$

\begin{abstract}
Background: Communication and information in order to reduce anxiety in the intensive care unit (ICU) has been described as area needing improvement. Therefore, the aim of this trial was to evaluate whether a structured information program that intensifies information given in standard care process reduces anxiety in ICU patients.

Methods: Multicenter, two-armed, non-blinded, parallel-group randomized controlled trial in hospitals in the cities of Marburg, Halle, and Stuttgart (Germany). The trial was performed in cardiac surgery, general surgery, and internal medicine ICUs. Two-hundred and eleven elective and non-elective ICU patients were enrolled in the study (intervention group, $n=104$; control group, $n=107$ ). The experimental intervention comprised a single episode of structured oral information that was given in addition to standard care and covered two main parts: (1) A more standardized part about predefined ICU specific aspects - mainly procedural, sensory and coping information, and (2) an individualized part about fears and questions of the patient. The control group received a non-specific episodic conversation of similar length additional to standard care. Both conversations took place at the beginning of the ICU stay and lasted 10-15 minutes. Study nurses administered both interventions. The primary outcome ICU-related anxiety (CINT-Score, 0-100 pts., higher scores indicate higher anxiety) was assessed after admission to a regular ward.

Results: The primary outcome could be measured in 82 intervention group participants and 90 control group participants resulting in mean values of 20.4 (SD 14.4) compared to 20.8 (SD 14.7) and a mean difference of -0.2 (Cl 95\% -4.5 to 4.1).

Conclusions: A structured information intervention additional to standard care during ICU stay had no demonstrated additional benefit compared to an unspecific communication of similar duration. Reduction of anxiety in ICU patients will probably require more continuous approaches to information giving and communication.
\end{abstract}

Trial registration: ClinicalTrials.gov NCT00764933.

Keywords: Intensive care units, Critical care, Anxiety, Prevention \& control, Nurse-patient relations, Information, Randomized controlled trial

\footnotetext{
* Correspondence: steffen.fleischer@medizin.uni-halle.de

${ }^{1}$ Institute of Health and Nursing Science, Medical Faculty,

Martin-Luther-University Halle-Wittenberg, Halle, Germany

Full list of author information is available at the end of the article
} 


\section{Background}

Qualitative and quantitative studies report patients' experience of intensive care unit (ICU) stay as highly stressful [1-5]. Psychological and physical stress in ICUs often is associated with higher levels of anxiety and feelings of uncertainty and helplessness [6,7]. Evidence suggests that such elevated and prolonged stress has negative consequences on health outcomes like delayed wound healing, susceptibility to respiratory infections, changed immune responses to vaccines [8], and is conceived as a risk factor of delirium [9]. Ineffective communication and lack of information further contribute to distress for patients [10-18]. International guidelines therefore emphasize the importance of effective communication for patient-centered care, especially with critically ill patients, but still there is little evidence on effective interventions $[19,20]$. This coincides with a recent Delphi study that rated priorities of ICU-related research in the domain of patient wellbeing [21]: Strategies to reduce stress and anxiety, and strategies to improve communication to meet patients' informational needs were identified as highly important [21].

Our literature search revealed only one randomized controlled trial which investigated a specific single episode information intervention in the ICU [22]. The investigators used audiotaped information after the patient's recovery from anesthesia. The results of this study suggest the effectiveness of a single episode situational information intervention to improve psychological parameters in the ICU. The effectiveness of information administered face-to-face to ICU patients in the ICU has not yet been investigated.

Therefore the aim of our trial was to evaluate whether a structured information program modeled as a brief single episode nursing care delivery of ICU-specific information via face-to-face communication compared to a non-specific verbal face-to-face communication of the same length contributes to a reduction of experienced anxiety in ICU-patients. Both interventions were supplementary to standard care and administered in the initial stage of treatment in an ICU [23].

\section{Methods}

\section{Trial design}

We conducted a prospective, multi-center, non-blinded, randomized controlled trial with two parallel groups in a 1:1 ratio. The study was registered with ClinicalTrials.gov as NCT00764933. Informed consent was obtained from all patients before inclusion in the study. The trial is reported in accordance with the CONSORT statement [24].

\section{Participants}

The study was performed between December 2007 and December 2009 in three hospitals in Germany (Marburg (coordinator) Halle, and Stuttgart) in cardiac surgery, general surgery, and medical ICUs. The study protocol [23] was approved by the respective ethics committees responsible for the study sites in the universities in Marburg, Halle, and Tübingen (Germany).

ICU patients in cardiac surgery, general surgery, and internal medicine (including local High Dependency Units) with scheduled and unscheduled ICU stays longer than 24 hours from enrollment were eligible for inclusion in our trial. All patients were recruited at the beginning of their ICU stay (meaning within the first 24 hours of consciousness post-admission). Exclusion criteria were: anticipated inability to fill in the mailed follow-up questionnaire, cognitive impairment, lack of German language ability, placed in a room with another study patient, more than 48 hours awake and clear in the ICU (because intervention was intended at the beginning of the ICU stay), or under the age of 18 .

Study nurses assessed cognitive impairment by means of the Richmond Agitation and Sedation Scale (RASS) [25] and the Confusion Assessment Method for the Intensive Care Unit (CAM-ICU) [26-28]. RASS values $<-3$ or a positive test of CAM-ICU (acute confusion: yes) were deemed as an inability to consent to study participation on the day of the assessment. Study nurses assessed these patients again on the following day. In this way it was possible for patients to be assessed more than once for study inclusion (temporary exclusion when patient was not able to give consent due to impaired consciousness). We only report the last-encountered reason for the exclusion of these patients.

\section{Interventions}

Effects of information interventions are often explained by the provision of individual relevant information and psychosocial support, making the situation more understandable and bearable for the patient. These explanations are concordant with Lazarus' cognitive-mediation theory of stress and emotion [29] which emphasizes the role of cognitive aspects (appraisal-reappraisal, emotion-focused coping, and emotional social support, tangible social support and informational social support) in the development of anxiety and stress. This framework also seems well-suited for an intervention rationale that encompasses giving information to all patients admitted to ICUs (i.e. including non-elective surgical patients or non-surgical patients). Therefore our study intervention applied Lazarus' cognitive-mediation theory of stress and emotion.

The experimental intervention was a single episode information intervention that included ICU-specific information and was implemented at an early stage of the patients' ICU stay to intensify information given in standard care process. We decided to develop and implement the experimental intervention as single episode information for two reasons: 
(1) The intervention should be pragmatic in regard to applicability and implementation fidelity. (2) Studies in regard to pre-operative information $[30,31]$ and Hwang et al. [22] successfully used single episode interventions. The intervention was designed as a guided conversation with both a standardized and an individualized part (Table 1). The standardized part of the intervention covered general information on nine topics (Table 2), which have been identified as relevant for patients in ICUs in previous studies [32-34]. All patients in the intervention group (IG) received this level of information. In the individual part the patients were allowed to choose any number of cards from seven that depicted common fears (Table 1). Complementary, the patients' needs for personal information on particular topics of the first part or additional topics were assessed by asking the patients if they wanted more detailed information of certain topics. These topics and the chosen cards (i.e. fears) were addressed in the subsequent individualized conversation. So IG patients had the opportunity to get sensory, procedural, and coping information on ICU-specific and additional topics, and could speak about their fears and anxieties.

The study investigators that were responsible for intervention development were all experienced within the field of intensive and anesthesia nursing care or clinical psychology. The nine topics and the seven fears on the cards were chosen in accordance with the research literature on pre-operative information and relieving aspects of ICU stay, and our own preliminary studies [34].

The control intervention was a non-specific conversation of the same length and was offered at the beginning of ICU stay as a semi-structured, self-directed, non-specific conversation (excluding information on the ICU stay) with a study nurse. Priority was given to topics that were related to overall health, family, occupational concerns, and recreation.

Both interventions were piloted in each study center to test feasibility and acceptability of both interventions.

A study nurse carried out the study procedure in each center, but none of the study nurses were involved in the standard care of the included patients. Treatment fidelity in the different centers was intended by comprehensive instruction of the study personnel for the implementation of the information and the control intervention. Study nurses documented length and content of the information and the control intervention. Thus, assessment of fidelity was done on a descriptive indirect level only. Additionally, the coordinating center in Marburg centrally monitored the trial by regular visits and clinical supervision in the participating study centers.

Both interventions took place immediately after recruitment, baseline measurement, and randomization and were supplementary to standard care.

\section{Outcomes}

All measurements and the timeline are summarized in Table 3.

\section{Primary outcome}

The primary outcome was the anxiety-related part (CINTScore) of the Questionnaire for Surgical ICU Patients (CINT questionnaire) on the experiences and the emotional state in the ICU that was recorded shortly after admission to a regular ward. This questionnaire has already been used in pilot studies $[32,33,35]$ and represents specific aspects of quality of life in relation to the ICU.

The CINT-Score covers experienced anxieties during the ICU stay and comprises the following items: fear of

Table 1 Structure of the experimental intervention and justification of its components

\begin{tabular}{|c|c|c|}
\hline $\begin{array}{l}\text { Part of the } \\
\text { intervention }\end{array}$ & Description & Justification \\
\hline I. Standardized part & $\begin{array}{l}\text { General information on nine relevant topics in the ICU } \\
\text { using a guideline (with examples for each topic) }\end{array}$ & $\begin{array}{l}\text { Topics were identified in trials of pre-operative information } \\
\text { and patient education. A guideline was chosen to achieve a } \\
\text { standardized structure but still having the possibility to make } \\
\text { setting and ICU specific adaptations. }\end{array}$ \\
\hline \multirow[t]{9}{*}{ II. Individualized part } & $\begin{array}{l}\text { Seven cards the patient could choose from, depicting } \\
\text { common fears associated with an ICU-stay: }\end{array}$ & \multirow{9}{*}{$\begin{array}{l}\text { The cards offered patients the opportunity to start a conversation } \\
\text { about specific fears, by helping them to articulate fear. So it was } \\
\text { possible for the trial staff to answer accordingly (e.g., What is being } \\
\text { done in the ICU to prevent this from happening to you?). Additionally } \\
\text { vocally impaired patients (e.g. by mechanical ventilation) could } \\
\text { indicate their fears, too. The opportunity to ask further questions } \\
\text { was to meet informational needs in patients that wanted to know } \\
\text { more or more detailed about their ICU stay. }\end{array}$} \\
\hline & 1. fear of complications & \\
\hline & 2. fear of suffocating & \\
\hline & 3. fear of pain & \\
\hline & 4. fear of being helpless & \\
\hline & 5. fear of death & \\
\hline & 6. fear of being lonely & \\
\hline & 7. fear of being confined & \\
\hline & $\begin{array}{l}\text { Opportunity to ask additional and detailed questions on the } \\
\text { ICU stay (recurring to the topics of part I or additional topics) }\end{array}$ & \\
\hline
\end{tabular}


Table 2 Topics of the standardized part of the experimental intervention

\begin{tabular}{|c|c|c|}
\hline Nr. & Topic & Details \\
\hline \multirow[t]{5}{*}{1} & People in the ICU & $\begin{array}{l}\text { - health care professionals (nurses } \\
\text { and intensive care nurses) }\end{array}$ \\
\hline & & - attending physician \\
\hline & & $\begin{array}{l}\text { - clothing, including specifics such } \\
\text { as masks, gloves etc. }\end{array}$ \\
\hline & & - change of shifts \\
\hline & & - ward rounds \\
\hline \multirow[t]{4}{*}{2} & Devices and & - monitor, including central monitoring \\
\hline & & - ventilator \\
\hline & & • infusion and syringe pump \\
\hline & & - alarms \\
\hline \multirow[t]{3}{*}{3} & Room furnishing & • clock \\
\hline & & - bell system \\
\hline & & • room size \\
\hline \multirow[t]{6}{*}{4} & Individual safety & $\begin{array}{l}\text { - tubes, drainages, wounds, urinary } \\
\text { catheters, fixation }\end{array}$ \\
\hline & & $\begin{array}{l}\text { - tube, respiratory mask, mechanical } \\
\text { ventilation }\end{array}$ \\
\hline & & - waking phase \\
\hline & & • intravenous access \\
\hline & & - bedding \\
\hline & & - dimming of the light \\
\hline \multirow[t]{4}{*}{5} & Schedule & - hospital stay duration \\
\hline & & - transfer to IMC \\
\hline & & - differences between IMC and ICU \\
\hline & & - nutrition \\
\hline \multirow[t]{2}{*}{6} & Communication & - nod, shake of the head (yes, no) \\
\hline & & - pens \\
\hline \multirow[t]{4}{*}{7} & Staff duties & - aspiration \\
\hline & & - mobilization \\
\hline & & - radiologic examinations \\
\hline & & - personal hygiene/oral hygiene \\
\hline \multirow[t]{3}{*}{8} & Conveniences & - pain medication \\
\hline & & - visiting hours \\
\hline & & $\begin{array}{l}\text { - information before nursing-medical } \\
\text { interventions }\end{array}$ \\
\hline \multirow[t]{3}{*}{9} & Helpful thoughts & $\begin{array}{l}\text { - "Everything is done for me. That is a } \\
\text { sign that everything worked alright". }\end{array}$ \\
\hline & & $\begin{array}{l}\text { - "I don't have to suffer from any pain; if } \\
\text { necessary I will receive additional medication. } \\
\text { In the meantime I can relax and continue to } \\
\text { breathe calmly". }\end{array}$ \\
\hline & & . "Only a little longer, then I have made it". \\
\hline
\end{tabular}

death, fear of severe suffering, fear of a handicap, fear of the future, fear of uncertainty, panic, strain, depression, loneliness, melancholy, lack of orientation, uncertainty, anger, optimism, and confidence. All items were rated on a 4-point scale from never to always. The final score was calculated as a Likert-scale, averaging the items (after inverting negative items) and transforming it to a scale of 0-100 (no anxiety to maximum anxiety). In an earlier study [35] the CINT-Score has shown good internal consistency (Cronbach's alpha $=0.88)$ (unpublished data).

\section{Secondary outcomes}

In addition to the retrospective CINT-Score a visual analogue scale supported by the Faces Anxiety Scale (FAS) [36] was used for measurements at $24 \mathrm{~h}$ and $48 \mathrm{~h}$ after intervention (ranges from $0-100$; no anxiety to maximum anxiety). The FAS has shown an acceptable level of validity in the ICU setting even for ventilated patients, while having a low respondent burden [36-39]. As a graphic representational scale, reliability can be assumed, too [40]. Additionally, the patients' level of consciousness and concentration regarding a potential ICU-related state of confusion (acute confusion: yes/no) was assessed with the CAM-ICU [26,27], including the RASS [25]. The CAM-ICU demonstrated good validity and reliability in ICU patients [41].

To compare anxiety levels after the ICU stay, the State Scale of the State and Trait Anxiety Inventory (STAI) [42] was recorded and calculated in accordance with the manual (ranges from 20-80). The STAI-State is a well-established measurement for anxiety with good validity, reliability, and has shown responsiveness to change [42]. This measure was transferred to a $0-100$ scale (no anxiety to maximum anxiety). The Visual Analogue ScaleAnxiety (VAS-A) [43] (ranges from 0-100; no anxiety to maximum anxiety) was measured in parallel to the STAIState. VAS-A has shown validity but data on reliability is limited [43].

ICU-related experiences were obtained with the CINT questionnaire (36 single items in relation to the categories: communication, physical state, environmental factors, and ICU-specific circumstances). For the analysis of additional effects the length of stay (ICU and hospital) were extracted from patients' records.

Three months after discharge a questionnaire was mailed to the participants of both study groups. We asked about health-related quality of life (QoL) using the Health Survey 12 Item Short Form (SF-12) [44], and individual QoL using the Schedule for Evaluation of Individual Quality of Life (SEIQoL) [45]. For this reason we developed and implemented a paper questionnaire version of the SEIQoL [46] which was used and tested for feasibility the first time in our study. SF-12 results were analyzed in accordance with the manual in the dimensions of the Mental Health Component Summary (SF-12 MCS) score and the Physical Health Component Summary (SF-12 PCS) score. The SF-12 has shown good reliability and 
Table 3 Measures in the course of the study, including baseline data $\left(t_{0}\right)$ and outcomes $\left(t_{1}-t_{5}\right)$

\begin{tabular}{|c|c|c|}
\hline & Point of measurement & Measurements \\
\hline$t_{0}$ & Day of enrolment (ICU) (before randomization) & $\begin{array}{l}\text { Socio-demographic data, routine treatment data, acute confusion } \\
\text { (CAM-ICU including RASS), anxiety state (FAS) }\end{array}$ \\
\hline$t_{1}$ & $24 \mathrm{~h}$ after study intervention & Acute confusion (CAM-ICU including RASS), anxiety state (FAS) \\
\hline$t_{2}$ & $48 \mathrm{~h}$ after study intervention & Acute confusion (CAM-ICU including RASS), anxiety state (FAS) \\
\hline$t_{3}$ & Admission to regular ward & $\begin{array}{l}\text { ICU related anxiety and experiences (CINT questionnaire including } \\
\text { CINT-Score), anxiety state (VAS-A, and STAI-State) }\end{array}$ \\
\hline $\mathrm{t}_{4}$ & Discharge from hospital & In-patient history and complications, length of stay and mode of discharge \\
\hline$t_{5}$ & 3 months after discharge (via post) & Individual quality of life (SEIQoL), health related quality of life (SF-12) \\
\hline
\end{tabular}

validity [47]. All QoL scores range from 0-100 (higher scores indicate better QoL).

\section{Sample size}

The sample size calculation was based on the CINT-Score as an anxiety related aspect of the ICU-patients' quality of life. This sum score is represented on a scale from 0 to 100 and was found to add up to a mean value of $M=28.0$ and a standard deviation of SD $=17.0$ in an earlier unpublished trial in an ICU population. On the notion that in QoL measures the differences within the scope of half a standard deviation are considered as noticeable [48] we determined a difference of 8.5 scale points to be clinically relevant. Further determining $\alpha=0.05$ and $\beta=0.20,70$ patients per group were needed to find this effect from a standard two-group $t$-test. The allowance of a $30 \%$ loss to follow-up resulted in an estimated sample size of $n=100$ per group, corresponding to a total sample size of $\mathrm{N}=200$ $(\mathrm{n}=100$ vs. $\mathrm{n}=100)$.

\section{Randomization}

A statistician computer generated the randomization sequence in advance employing a stratified (by center and clinical department) balanced block randomization. A study nurse employed for the trial randomized participants individually. Group allocation was concealed by using sequentially numbered opaque sealed envelopes and took place right after informed consent was received and baseline assessment. Contamination was avoided by restricting recruitment to one patient per room in the ICU at the same time.

\section{Statistical methods}

All data was double entered using double entry verification provided by EpiData Data Entry [49]. Data analysis was performed using the statistical software R [50]. Final data analysis was done in accordance with the published study protocol [23].

Analysis of the primary endpoint used an ANCOVA model with the primary endpoint as the response, and the center as a covariate, thus allowing for the stratified randomization procedure. The treatment effect was calcu- lated as an adjusted difference in means with a corresponding 95\% confidence interval (CI 95\%), $\alpha$ was set to 0.05 .

Secondary endpoints were assessed with the respective adjusted models, using standard ANCOVA models for continuous outcomes. Mixed effects models were used for repeatedly measured outcomes to adjust for within-patient correlations [51]. Secondary endpoints analyses were considered as merely exploratory to avoid alpha-inflation.

The analyses of all outcomes were by intention to treat (all patients analyzed as they were randomized). However, no imputation of missing data was performed, and we did complete cases analyses. Missing data for single measures was handled in accordance with the respective measure's manual.

\section{Results}

A total of 1838 ICU patients were assessed for eligibility. Figure 1 displays the reasons for study exclusion. The three main reasons for study exclusion were unable to give consent, longer than $48 h$ fully conscious, and expected ICU stay shorter than $24 \mathrm{~h}$. We recruited 211 patients with 92 patients in Marburg, 59 patients in Halle, and 60 patients in Stuttgart. Randomization resulted in 104 intervention group participants and 107 control group participants. Two patients in the control group received the information intervention as they insisted on it after randomization, but were analyzed in the control group.

Baseline characteristics are displayed in Table 4. There were no clinically relevant differences at baseline except for baseline anxiety measured with the FAS. We conducted sensitivity analyses controlling for FAS at $t_{0}$ and compared these analyses with our predefined analyses.

\section{Intervention fidelity}

Data on implementation of the interventions in both groups are presented in Table 5. Intervention length differed significantly between the two study groups (IG mean: 13.5 minutes (SD 3.6) vs. CG mean 11.3 minutes (SD 3.9), mean difference $=2.2$ minutes, CI 95\% $(1.2 ; 3.2)$, $\mathrm{p}<0.001)$.

Of the patients that received the experimental intervention ( $n=106$, IG patients plus the two CG patients 


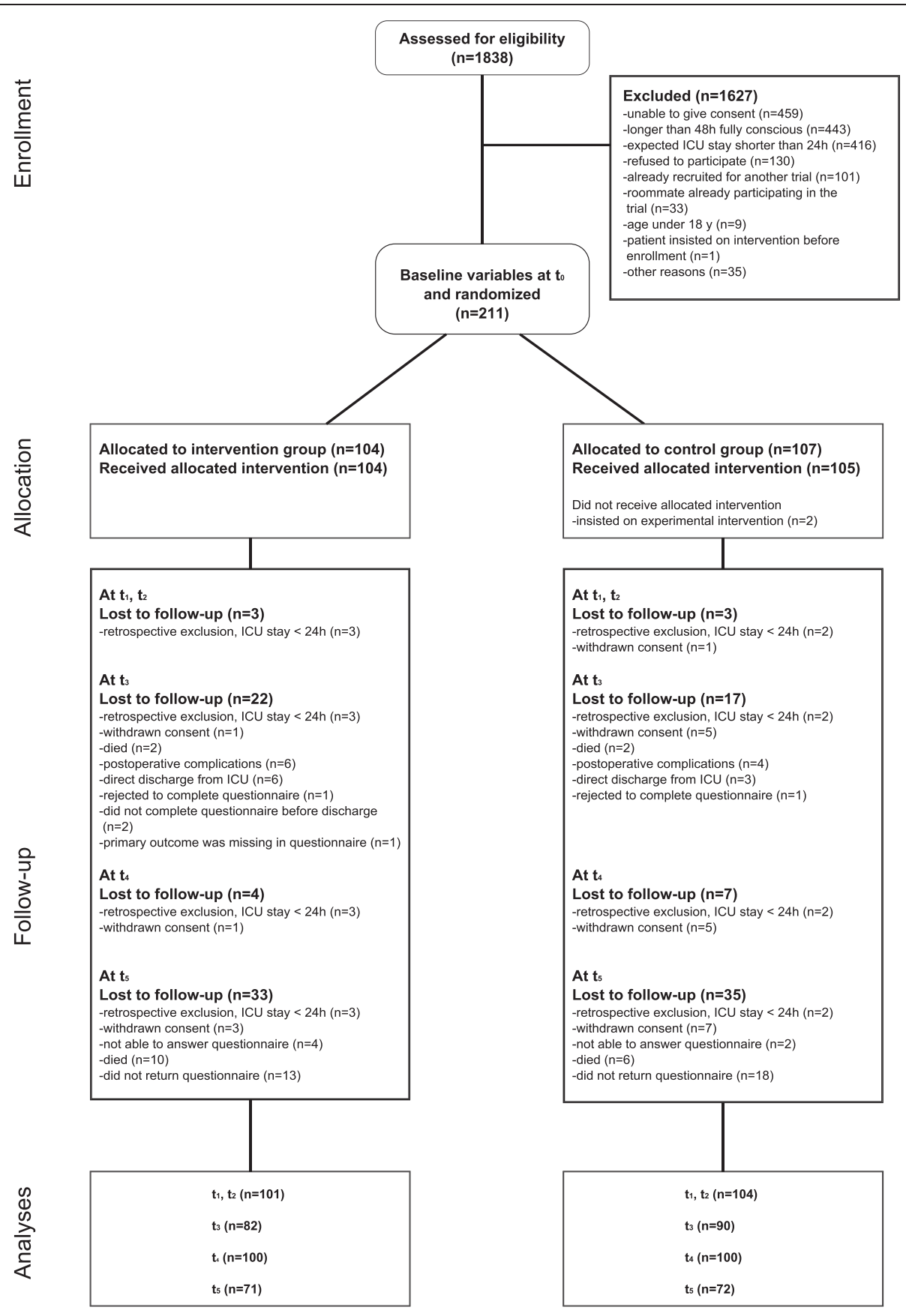

Figure 1 Flow of participants through the trial.

that received the experimental intervention), 61 patients (57.5\%) chose at least one fear-depicting card presented to them. Overall 124 cards were drawn (multiple cards per person were possible). The three most frequent fears were: fear of complications ( $\mathrm{n}=34$ times drawn), fear of suffocating ( $\mathrm{n}=27$ times drawn), and fear of pain $(\mathrm{n}=25$ times drawn). Forty-five patients (42.5\%) did not choose any of the cards presented to them. In regard to the ICU-specific topics, 74 patients $(69.8 \%)$ that received the experimental intervention ( $\mathrm{n}=106$, IG patients plus the two CG patients that received the experimental intervention) asked for more detailed information on the topics of the first conversation part and/or other topics: resulting in 327 requests on the topics; multiple requests per person possible. The three most frequent topics were: devices and monitoring ( $\mathrm{n}=55$ times asked), room furnishing ( $\mathrm{n}=47$ times asked), and people in the ICU ( $\mathrm{n}=44$ times asked). Thirty-two patients $(30.2 \%)$ did not 
Table 4 Baseline characteristics of the study population

\begin{tabular}{|c|c|c|}
\hline Variable & $\begin{array}{l}\text { Intervention } \\
\text { group }(n=104)\end{array}$ & $\begin{array}{l}\text { Control group } \\
(n=107)\end{array}$ \\
\hline \multicolumn{3}{|l|}{ Sex, n (\%) } \\
\hline Men & $66(63.5)$ & $71(66.4)$ \\
\hline Women & $38(36.5)$ & 36 (33.6) \\
\hline \multicolumn{3}{|l|}{ Age, years } \\
\hline Mean (SD) & $63.3(14.5)$ & $65.8(11.8)$ \\
\hline Median (IQR) & $68.0(55.0-73.0)$ & $68.0(59.0-74.0)$ \\
\hline \multicolumn{3}{|l|}{ Department, n (\%) } \\
\hline Cardiac surgery patients & $52(50.0)$ & $50(46.7)$ \\
\hline General surgery patients & $19(18.3)$ & $22(20.6)$ \\
\hline Medical patients & $33(31.7)$ & $35(32.7)$ \\
\hline \multicolumn{3}{|l|}{ Main procedure, $\mathrm{n}(\%)$} \\
\hline Surgical intervention & $71(68.3)$ & $79(73.8)$ \\
\hline Non-surgical intervention & $21(20.2)$ & $22(20.6)$ \\
\hline Diagnostic measure & $10(9.6)$ & $3(2.8)$ \\
\hline Other procedure & $2(1.9)$ & $3(2.8)$ \\
\hline \multicolumn{3}{|l|}{$\begin{array}{l}\text { ICD-10 chapter, main } \\
\text { diagnosis, } \mathrm{n}(\%)\end{array}$} \\
\hline Diseases of the circulatory system & $70(67.3)$ & $82(76.6)$ \\
\hline Neoplasms & $5(4.8)$ & $8(7.5)$ \\
\hline Diseases of the digestive system & $5(4.8)$ & $6(5.6)$ \\
\hline Diseases of the respiratory system & $7(6.7)$ & $1(0.9)$ \\
\hline $\begin{array}{l}\text { Injury, poisoning and certain other } \\
\text { consequences of external causes }\end{array}$ & $4(3.8)$ & $4(3.7)$ \\
\hline $\begin{array}{l}\text { Congenital malformations, deformations, } \\
\text { and chromosomal abnormalities }\end{array}$ & $4(3.8)$ & $1(0.9)$ \\
\hline Other chapters ${ }^{a}$ & $9(8.7)$ & $5(4.7)$ \\
\hline \multicolumn{3}{|l|}{$\mathrm{FAS}^{b}\left(\mathrm{t}_{0}\right)$} \\
\hline Mean (SD) & $24.5(18.9)$ & $30.2(22.1)$ \\
\hline Median (IQR) & $20.0(10.0-37.5)$ & $25.0(10.0-48.8)$ \\
\hline \multicolumn{3}{|l|}{ SAPS $\|^{C}\left(t_{0}\right)$} \\
\hline Mean (SD) & $23.8(8.2)$ & $26.1(10.2)$ \\
\hline Median (IQR) & $23.0(18.0-29.0)$ & $24.5(20.0-31.0)$ \\
\hline \multicolumn{3}{|l|}{ TISS $28^{d}\left(\mathrm{t}_{0}\right)$} \\
\hline Mean (SD) & $10.6(5.6)$ & $11.5(6.5)$ \\
\hline Median (IQR) & $10.0(5.0-14.0)$ & $10.0(10.0-14.0)$ \\
\hline \multicolumn{3}{|l|}{$\begin{array}{l}\text { Type of ventilation in intensive } \\
\text { care unit, } \mathrm{n}(\%)\end{array}$} \\
\hline No ventilation & $34(32.7)$ & $40(37.4)$ \\
\hline Non-invasive ventilation & $8(7.7)$ & $2(1.9)$ \\
\hline Invasive ventilation & $62(59.6)$ & $65(60.7)$ \\
\hline
\end{tabular}

Table 4 Baseline characteristics of the study population (Continued)

Ventilation in intensive care unit before the intervention, hours ${ }^{e}$

Mean (SD) $\quad 25.0(46.6) \quad 41.8(127.2)$

Median (IQR) $10.3(6.0-18.6) \quad 10.0(7.0-20.0)$

${ }^{a}$ Summarizes all other ICD-10 chapters with less than five patients in total; ${ }^{b}$ ranges from 0 (no anxiety) to 100 (maximal anxiety); ' higher score means worse overall health; ${ }^{d}$ higher score means higher intervention needs; ${ }^{e}$ only ventilated patients, before interventions.

ask for further information on the topics of the first conversation part or additional topics.

The main conversation topics (only the main topic of conversation was documented) in the control group ( $\mathrm{n}=105$, CG patients minus the two CG patients that received the experimental intervention) were: overall health status $(\mathrm{n}=40)$, family $(\mathrm{n}=39)$, recreation $(\mathrm{n}=11)$, occupation $(\mathrm{n}=11)$, and other topics $(\mathrm{n}=4)$.

\section{Follow-up}

For analyses of repeated measurements $\left(t_{1}, t_{2}\right) 205$ patients (97\%) were eligible. One-hundred and seventy-two patients $(82 \%)$ could be included for the analysis of the primary outcome $\left(t_{3}\right)$. The mailed final questionnaire was answered by 143 patients $(68 \%)\left(t_{5}\right)$. Reasons for incomplete data are presented in Figure 1. There were no differences, either in numbers or reasons for loss to follow-up, between the two study groups.

In order to check similarity of IG and CG after drop out we compared baseline values of the complete cases for the primary outcome. Overall patients with a higher FAS, SAPS II, and TISS 28 were more likely to be lost to

Table 5 Circumstances under which the interventions were applied

\begin{tabular}{lll}
\hline Variable & $\begin{array}{l}\text { Intervention group } \\
(\mathbf{n = 1 0 4 )}\end{array}$ & $\begin{array}{l}\text { Control group } \\
(\mathbf{n}=107)\end{array}$ \\
\hline Duration of intervention, minutes & $13.5(3.6)$ & $11.3(3.9)$ \\
Mean (SD) & $15.0(10.0-15.0)$ & $10.0(10.0-14.0)$ \\
Median (IQR) & & \\
Day of intensive care unit stay & $1.1(1.6)$ & $1.7(3.1)$ \\
Mean (SD) & $1.0(0.0-1.0)$ & $1.0(1.0-1.0)$ \\
Median (IQR) & & \\
Ventilated during intervention, & & $7(6.5)$ \\
n (\%) & $5(4.8)$ & $100(93.5)$ \\
Yes & $99(95.2)$ & \\
No & & $3(2.8)$ \\
Family members present, $n(\%)$ & $5(4.8)$ & $104(97.2)$ \\
Yes & $99(95.2)$ & \\
No & & \\
\hline
\end{tabular}


follow-up in our sample but characteristics still were evenly distributed between the IG and the CG.

\section{Outcomes}

Adjusted mean differences (adj. MD) and related 95\% CIs are presented in Figure 2. There were no significant and no clinical relevant differences between the two groups for the primary and secondary outcomes. A sensitivity analysis with FAS $t_{0}$ as an additional covariate did not show different results; therefore only results from the model with study center as a covariate are presented. The raw means without adjustment are presented in Table 6.

The treatment effect as a mean difference with regard to the repeated measurement of the FAS $\left(t_{0}\right.$ through $\left.t_{2}\right)$ was calculated as a fixed effect of a mixed effects model with a random intercept. Sensitivity analyses with and without FAS $t_{0}$ as a covariate showed considerable influence of baseline anxiety. The fixed effect was -7.8 , 95\% CI -15.7 to $0.1, p=0.05$, in favor of the IG when not controlling for FAS $t_{0}$. This effect changed to -2.4 , 95\% CI -6.4 to $1.5, \mathrm{p}=0.14$, in favor of the IG when controlling for FAS $t_{0}$. Thus, our preplanned analysis (Model 1) for this secondary outcome does not safely exclude the possibility of a clinically relevant difference between the two groups while an analysis that controls for baseline FAS (Model 2) and its related CI 95\% makes a clinically relevant difference in future research rather unlikely.
Therefore we favor Model 2 as it is more conservative to the null hypothesis.

In regard to the prevalence of acute confusion in the ICU assessed with the CAM-ICU ( $t_{0}$ through $\left.t_{2}\right)$ a mixed effects logistic regression model (Generalized Linear Mixed Model with Penalized Quasi-Likelihood Estimation) using our basic model did not converge. Therefore we used a binomial Generalized Linear Model without random effects to get an impression of intervention effects. The comparison of IG vs. CG revealed an odds ratio of 0.33 , $95 \%$ CI 0.05 to $1.49, \mathrm{p}=0.18$, to suffer from acute confusion in favor of the IG ( $t_{0}$ IG: 0/104, CG: 0/107; $t_{1}$ IG: 2/96, CG: 6/100; $t_{2}$ IG: 0/70, CG: 5/72).

Mean length of ICU stay was 4.3 (SD 4.5) days in the IG vs. 4.9 (SD 5.5) days in the CG with a mean difference adjusted for study center of -0.4 days in favor of the IG, $95 \% \mathrm{CI}-1.7$ to $0.9, \mathrm{p}=0.56$. Overall mean length of hospital stay was 15.4 (SD 13.8) days in the IG vs. 16.5 (SD 13.5) days in the CG with a mean difference adjusted for study center of -1.0 days in favor of the IG, $95 \%$ CI -4.7 to $2.7, \mathrm{p}=0.59$.

On the item level of the CINT-Score and the CINT questionnaire no significant group differences could be found. We did not predefine any adverse events as we did not expect negative effects of both study interventions.

\section{Discussion}

This is the first trial that tested a personal single episode information intervention in ICU patients. Our trial failed

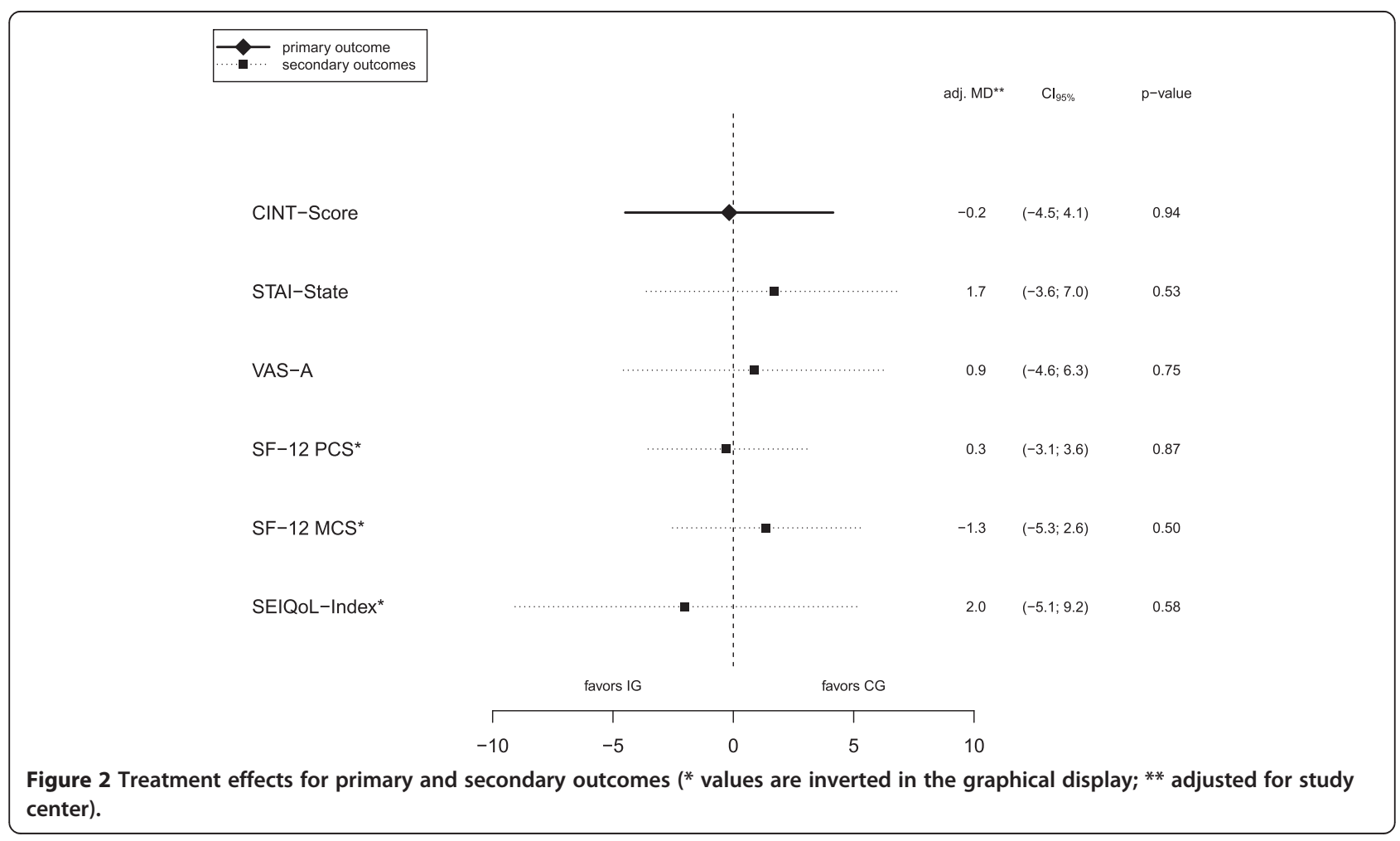


Table 6 Results of the primary and secondary outcomes

\begin{tabular}{lcl}
\hline & IG Mean (SD) & CG Mean (SD) \\
\hline $\mathrm{t}_{3}$ measurements: admission to regular ward & \\
CINT-Score $^{a}$ & $20.4(14.4)$ & $20.8(14.7)$ \\
STAI-State (transformed) $^{a}$ & $33.0(17.0)$ & $32.1(19.1)$ \\
STAI-State (original) $^{b}$ & $40.0(10.4)$ & $39.2(11.1)$ \\
VAS-A $^{a}$ & $12.7(18.6)$ & $11.9(17.4)$ \\
$\mathrm{t}_{5}$ measurements: 3 months after discharge & \\
SF-12 PCS $^{c}$ & $40.6(9.4)$ & $40.4(10.0)$ \\
SF-12 MCS $^{c}$ & $46.9(11.3)$ & $48.2(11.2)$ \\
SEIQoL-Index $^{c}$ & $74.9(18.2)$ & $73.6(20.1)$ \\
\hline
\end{tabular}

Cases with complete data: CINT-Score (IG/CG 82/90); STAI-State (79/89); VAS-A (81/88); SF-12 PCS (64/66); SF-12 MCS (64/66); SEIQoL-Index (58/60); ${ }^{a}$ ranges from 0 (no anxiety) to 100 (maximum anxiety); ${ }^{b}$ ranges from 20 (no anxiety) to 80 (maximum anxiety); ' ranges from 0 to 100 , higher scores mean higher quality of life.

Values are unadjusted mean values (standard deviation).

to demonstrate a significant decrease of anxiety measured with the CINT-Score in the IG. We found no benefit for an ICU-specific single episode information intervention in the ICU compared to an unspecific conversation of comparable length. This result is the opposite of former studies that investigated single episode information given preoperatively $[30,31]$ or to the findings of Hwang et al. [22]. For some secondary outcomes (length of ICU stay, length of hospital stay, and prevalence of acute confusion) there may be a signal for improvement. However these improvements must be interpreted with caution as there was too much statistical uncertainty.

Our study had certain limitations. We cannot exclude a reduced effect of an information intervention due to patients' limited memory, and thus information processing ability [52]. However, our recruitment and screening procedure by means of RASS and CAM-ICU most likely lead to an underrepresentation of confused or cognitively impaired patients in our sample. Study personnel and participants were not blinded to allocation after randomization. Although we do not expect a high risk of performance bias since the investigators were not involved in patient care and co-interventions can be excluded. Measures of treatment effect used in this study mainly included patient reported outcomes and no physiologic measures like psycho-endocrine stress indicators, which are highly associated with anxiety [53]. Psycho-endocrine stress indicators may represent a more objective method to measure emotional distress than a one-item scale like the FAS or a retrospective questionnaire like the CINT-Score. However, the validity of these indicators could be compromised by applied drugs and health status of the participants in the ICU [54]. FAS and CINT-Score values indicate rather low levels of anxiety in our study sample (Table 6). This corresponds to the high proportion $(42.5 \%)$ of patients that did not select a fear card in the IG, indicating they saw no need for further communication on fears. A further explanation could be the recruitment procedure, as our sample had an overrepresentation of "healthier" ICU-patients than is generally found in patients in high dependency units or intermediate care wards. Consequently, a reduction of an already low level of anxiety seems rather unlikely. It has to be questioned whether anxiety is a relevant outcome for information interventions in cognitive unimpaired ICU patients or if stereotyped information actually can have positive effects compared to more patient centered communication approaches. Nevertheless, a certain proportion of ICU patients suffered considerably from fear and anxiety. Informational needs seem to vary widely as indicated by the large variability of chosen cards and requested information topics in our study. Approaches that encompass more continuous information giving and patient centered communication, i. e. interventions like staff education on communication and patient information may be more promising to investigate.

The measurement of the Therapeutic Intervention Scoring System (TISS 28) [55] and Simplified Acute Physiology Score (SAPS II) [56] at baseline indicate that our study population compares well to ICU patients in other studies. While the mean TISS 28 in the original validation study [55] was substantially higher than in our study, meaning we had included a sample with considerably fewer care activities than the former validation sample. The same holds true for the severity of illness measured with the SAPS II. Most studies that used the SAPS II [57-59] showed a higher mean score (i.e. higher severity of illness) than our study sample. The lower TISS 28 and SAPS II scores of our study are correspond with the low ICU related mortality (mortality rate until $t_{3}$ ) in our study sample (IG: 2/104; CG: 2/107). These indicators for a positively selected sample from the ICU population are further reinforced by the low prevalence of patients with acute confusion compared to $17 \%$ for cardiac surgery patients in other studies [60]. This overall positive selection of our study sample can mainly be explained by our inclusion criteria, specifically due to the absence of a cognitive impairment as a prerequisite for informed consent and enrolment in our study.

Our study had also strengths. To avoid an intervention effect simply due to increased personal attention we implemented optimized usual care through unspecific conversation of the same length as a control intervention instead of a null intervention. Thus, the comparator investigated in our study is more rigorous than the one used in the study by Hwang et al. [22] where an intervention effect due to increased attention cannot be safely excluded. Although the length of the two interventions in the IG and CG differed significantly we rated the difference as clinically 
none-relevant. As indicated by Table 5, we were able to achieve good fidelity to our study protocol [23] for both groups and to perform the interventions in an early stage of the ICU stay. In regard to a difficult study population we were able to achieve our preplanned sample size. We published our study protocol and registered our study. No deviation from our study protocol occurred.

\section{Conclusions}

We conclude that a single episode information intervention in the ICU has no benefit to cognitively unimpaired patients with a low intervention profile (i.e. low scores in TISS 28 and SAPS II) compared to a nonspecific personal conversation. Therefore, single episode structured information for patients early in their ICU stay cannot be recommended for routine use. Approaches that try to improve staff communication skills in general and provide information during the whole ICU stay should be investigated in future ICU communication and information research.

\section{Key messages}

- A single episode information intervention during ICU stay does not reduce anxiety in patients compared to an unspecific conversation of similar length.

- More intensified approaches that emphasize improvement of nurse-patient communication and information throughout the entire stay might be more successful and should be targeted in future studies.

\begin{abstract}
Abbreviations
ANCOVA: Analysis of covariance; CAM-ICU: Confusion assessment method for the intensive care unit; CG: Control group; CINT: Fragebogen chirurgische intensivstation (questionnaire for surgical ICU patients); CINT-Score: Anxiety-related part of the CINT; FAS: Faces anxiety scale; ICU: Intensive care unit; IG: Intervention group; M: Mean; QoL: Quality of life; RASS: Richmond agitation and sedation scale; SAPS II: Simplified acute physiology score; SD: Standard deviation; SEIQoL: Schedule for evaluation of Individual quality of life; SF-12: Health survey 12 item short form; SF-12 MCS: Mental health component summary of the SF-12; SF-12 PCS: Physical health component summary; STAl: State and trait anxiety inventory; TISS 28: Therapeutic intervention scoring system; VAS-A: Visual analogue scale-anxiety.
\end{abstract}

\section{Competing interests}

The authors declare that they have no competing interests.

\section{Authors' contributions}

SF wrote the first draft of the manuscript. All authors contributed equally to the final manuscript and made substantial intellectual contributions. AB, SF, and TRN had the idea for the trial rationale and initiated the trial proposal. All authors were responsible for conception and design of the final study protocol. TRN was the responsible coordinator of the study center in Marburg, AH in Stuttgart, and JB in Halle. RB monitored all participating study centers in regard to compliance to the study protocol. RB, TRN, AH, SF, and $A B$ developed the study intervention. OK planned the statistical analysis and randomization. RB and SF supervised data entry. OK and SF conducted the statistical analysis. All authors read and approved the final manuscript.

\section{Acknowledgements}

The trial was funded by the German Federal Ministry of Education and Research (BMBF grant 01 GT0601 and 01GT0603 as a project in the Nursing Research Network "Middle-South").

\section{Author details}

'Institute of Health and Nursing Science, Medical Faculty,

Martin-Luther-University Halle-Wittenberg, Halle, Germany. ${ }^{2}$ Institute of Medical Epidemiology, Biostatistics, and Informatics, Medical Faculty, Martin-Luther-University Halle-Wittenberg, Halle, Germany. ${ }^{3}$ Städtisches Klinikum München GmbH Akademie, Munich, Germany. ${ }^{4}$ Sana Herzchirurgische Klinik Stuttgart, Stuttgart, Germany. ${ }^{5}$ Department 4: Health and Social Work, University of Applied Sciences, Frankfurt/Main, Germany.

${ }^{6}$ Hessian Institute of Nursing Research (HessIP), Franfurt/Main, Germany. ${ }^{7}$ Department of Nursing Research, University Hospital Giessen and Marburg, Location Marburg, Germany. ${ }^{8}$ Institute for Theoretical Surgery/Department of Visceral, Thoracic and Vascular Surgery, University Hospital Giessen and Marburg, Location Marburg, Germany.

Received: 23 September 2013 Accepted: 17 June 2014 Published: 28 June 2014

\section{References}

1. Hofhuis JG, Spronk PE, van Stel HF, Schrijvers AJ, Rommes JH, Bakker J: Experiences of critically ill patients in the ICU. Intensive Crit Care Nurs 2008, 24(5):300-313.

2. Löf $L$, Berggren $L$, Ahlström G: ICU patients' recall of emotional reactions in the trajectory from falling critically ill to hospital discharge: follow-ups after 3 and 12 months. Intensive Crit Care Nurs 2008, 24(2):108-121.

3. Samuelson KAM, Lundberg D, Fridlund B: Light vs. heavy sedation during mechanical ventilation after oesophagectomy - a pilot experimental study focusing on memory. Acta Anaesthesiol Scand 2008, 52(8):1116-1123.

4. Engström A, Grip K, Hamrén M: Experiences of intensive care unit diaries: 'touching a tender wound'. Nurs Crit Care 2009, 14(2):61-67.

5. Wang K, Zhang B, Li C, Wang C: Qualitative analysis of patients' intensive care experience during mechanical ventilation. J Clin Nurs 2009, 18(2):183-190.

6. Stein-Parbury J, McKinley S: Patients' experiences of being in an intensive care unit: a select literature review. Am J Crit Care 2000, 9(1):20-27.

7. McKinley S, Nagy S, Stein-Parbury J, Bramwell M, Hudson J: Vulnerability and security in seriously ill patients in intensive care. Intensive Crit Care Nurs 2002, 18(1):27-36.

8. Vileikyte L: Stress and wound healing. Clin Dermatol 2007, 25(1):49-55.

9. Arend $\mathrm{E}$, Christensen M: Delirium in the intensive care unit: a review. Nurs Crit Care 2009, 14(3):145-154.

10. Salyer J, Stuart BJ: Nurse-patient interaction in the intensive care unit. Heart Lung 1985, 14(1):20-24.

11. Rundell S: A study of nurse-patient interaction in a high dependency unit. Intensive Care Nurs 1991, 7(3):171-178.

12. Turnock C: Communicating with patients in ICU. Nurs Stand 1991, 5(15):38-40.

13. Hagland MR: Nurse-patient communication in intensive care: a low priority? Intensive Crit Care Nurs 1995, 11(2):111-115.

14. Fowler SB: Impaired verbal communication during short-term oral intubation. Nurs Diagn 1997, 8(3):93-98.

15. Llenore E, Ogle KR: Nurse-patient communication in the intensive care unit: a review of the literature. Aust Crit Care 1999, 12(4):142-145.

16. Wojnicki-Johansson G: Communication between nurse and patient during ventilator treatment: patient reports and RN evaluations. Intensive Crit Care Nurs 2001, 17(1):29-39.

17. Alasad J, Ahmad M: Communication with critically ill patients. J Adv Nurs 2005, 50(4):356-362.

18. Magnus VS, Turkington L: Communication interaction in ICU - patient and staff experiences and perceptions. Intensive Crit Care Nurs 2006, 22(3):167-180.

19. McCauley K, Irwin RS: Changing the work environment in intensive care units to achieve patient-focused care: the time has come. Am J Crit Care 2006, 15(6):541-548

20. National Institute for Health and Care Excellence: Acutely III Patients in Hospital. Recognition of and Response to Acute Illness in Adults in Hospital CG50. London: National Institute for Health and Care Excellence; 2007:6.

21. Blackwood B, Albarran JW, Latour JM: Research priorities of adult intensive care nurses in 20 European countries: a Delphi study. J Adv Nurs 2011, 67(3):550-562.

22. Hwang SL, Chang Y, Ko WJ, Lee MB: Stress-reducing effect of physician's tape-recorded support on cardiac surgical patients in the intensive care unit. J Formos Med Assoc 1998, 97(3):191-196.

23. Fleischer S, Berg A, Neubert TR, Koller M, Behrens J, Becker R, Horbach A, Radke J, Rothmund M, Kuss O: Structured information during the ICU stay 
to reduce anxiety: study protocol of a multicenter randomized controlled trial. Trials 2009, 10(84):1-9.

24. Moher D, Hopewell S, Schulz KF, Montori V, Gotzsche PC, Devereaux PJ, Elbourne D, Egger M, Altman DG: CONSORT 2010 explanation and elaboration: updated guidelines for reporting parallel group randomised trials. BMJ 2010, 340:c869.

25. Sessler CN, Gosnell MS, Grap MJ, Brophy GM, O'Neal PV, Keane KA, Tesoro EP, Elswick RK: The Richmond agitation-sedation scale: validity and reliability in adult intensive care unit patients. Am J Respir Crit Care Med 2002, 166(10):1338-1344

26. Ely EW, Inouye SK, Bernard GR, Gordon S, Francis J, May L, Truman B, Speroff T, Gautam S, Margolin R, Hart RP, Dittus R: Delirium in mechanically ventilated patients: validity and reliability of the confusion assessment method for the intensive care unit (CAM-ICU). JAMA 2001, 286(21):2703-2710.

27. Ely EW, Margolin R, Francis J, May L, Truman B, Dittus R, Speroff T, Gautam S, Bernard GR, Inouye SK: Evaluation of delirium in critically ill patients: validation of the confusion assessment method for the intensive care unit (CAM-ICU). Crit Care Med 2001, 29(7):1370-1379.

28. Ely EW, Truman B: The Confusion Assessment Method for the ICU (CAM-ICU). Training Manual. Nashville, TN: MPH and Vanderbilt University; 2002:1-12.

29. Lazarus RS: Stress And Emotion: A New Synthesis. New York: Springer; 1999:196.

30. Shuldham CM, Fleming S, Goodman H: The impact of pre-operative education on recovery following coronary artery bypass surgery. A randomized controlled clinical trial. Eur Heart J 2002, 23(8):666-674.

31. Scott A: Managing anxiety in ICU patients: the role of pre-operative information provision. Nurs Crit Care 2004, 9(2):72-79.

32. Bohrer T, Koller M, Neubert T, Moldzio A, Beaujean O, Hellinger A, Lorenz W, Rothmund M: Wie erleben allgemeinchirurgische Patienten die Intensivstation? Ergebnisse einer prospektiven Beobachtungsstudie. Chirurg 2002, 73(5):443-450.

33. Neubert TR, Koller M, Bohrer T: Wie erleben Patienten den Aufenthalt auf einer chirurgischen Intensivstation? Eine prospektive Beobachtungsstudie aus Sicht der Pflege. Intensiv 2004, 12:120-129.

34. Berg A, Fleischer S, Neubert TR, Koller M: Entwicklung und Pretest eines Programms mit spezifischen Informationen zum Intensivstationsaufenthalt. Hallesche Beiträge zu den Gesundheits- und Pflegewissenschaften 2004, 3(3):18-20.

35. Berg A, Fleischer S, Koller M, Neubert TR: Preoperative information for ICU patients to reduce anxiety during and after the ICU-stay: protocol of a randomized controlled trial [NCT00151554]. BMC Nurs 2006, 5(4):1-7.

36. McKinley S, Coote K, Stein-Parbury J: Development and testing of a faces scale for the assessment of anxiety in critically ill patients. J Adv Nurs 2003, 41(1):73-79.

37. McKinley S, Madronio C: Validity of the faces anxiety scale for the assessment of state anxiety in intensive care patients not receiving mechanical ventilation. J Psychosom Res 2008, 64(5):503-507.

38. Gustad LT, Chaboyer W, Wallis M: Performance of the faces anxiety scale in patients transferred from the ICU. Intensive Crit Care Nurs 2005, 21(6):355-360.

39. McKinley S, Stein-Parbury J, Chehelnabi A, Lovas J: Assessment of anxiety in intensive care patients by using the faces anxiety scale. Am J Crit Care 2004, 13(2):146-152.

40. Patrician PA: Single-item graphic representational scales. Nurs Res 2004 53(5):347-352.

41. Schuurmans MJ, Deschamps PI, Markham SW, Shortridge-Baggett LM, Duursma SA: The measurement of delirium: review of scales. Res Theory Nurs Pract 2003, 17(3):207-224.

42. Laux L, Glanzmann P, Schaffner P, Spielberger CD: Das State-Trait-Angstinventar. Theoretische Grundlagen und Handanweisung. Weinheim: Beltz-Testges; 1981:1-76.

43. Chlan LL: Relationship between two anxiety instruments in patients receiving mechanical ventilatory support. J Adv Nurs 2004, 48(5):493-499.

44. Bullinger M, Kirchberger I: SF-36: Fragebogen zum Gesundheitszustand: Handanweisung. Göttingen: Hogrefe; 1998:65-73.

45. Hickey AM, Bury G, O'Boyle CA, Bradley F, O'Kelly FD, Shannon W: A new short form individual quality of life measure (SEIQoL-DW): application in a cohort of individuals with HIV/AIDS. BMJ 1996, 313(7048):29-33.

46. Becker R, Berg A, Fleischer S, Neubert TR: Messung patientengenerierter Lebensqualität mittels schriftlicher Befragung. Entwicklung und erste Ergebnisse in der schriftlichen Anwendung des SEIQoL. Hallesche Beiträge zu den Gesundheits- und Pflegewissenschaften 2009, 8(4):1-15.

47. Ware J Jr, Kosinski M, Keller SD: A 12-item short-form health survey: construction of scales and preliminary tests of reliability and validity. Med Care 1996, 34(3):220-233.
48. Norman GR, Sloan JA, Wyrwich KW: Interpretation of changes in health-related quality of life: the remarkable universality of half a standard deviation. Med Care 2003, 41(5):582-592

49. Lauritsen JM: EpiData Data Entry, Data Management and Basic Statistical Analysis System. Odense: EpiData Association; 2008.

50. R Development Core Team: R: A Language And Environment For Statistical Computing. Vienna: R Foundation for Statistical Computing; 2010.

51. Brown H, Prescott R: Applied Mixed Models in Medicine. New York: Wiley; 2006:215-231

52. Jones $C$, Griffiths RD, Humphris G: Disturbed memory and amnesia related to intensive care. Memory 2000, 8(2):79-94.

53. Hubert W, Möller M, Nieschlag E: Stress reactions in response to the procedure of LHRH tests as measured by salivary and serum cortisol and psychological variables. Horm Res 1989, 32(5-6):198-202

54. Cooper MS, Stewart PM: Corticosteroid insufficiency in acutely ill patients. N Engl J Med 2003, 348(8):727-734.

55. Miranda DR, de Rijk A, Schaufeli W: Simplified therapeutic intervention scoring system: the TISS-28 items-results from a multicenter study. Crit Care Med 1996, 24(1):64-73.

56. Le Gall JR, Lemeshow S, Saulnier F: A new simplified acute physiology score (SAPS II) based on a European/North American multicenter study. JAMA 1993, 270(24):2957-2963.

57. Agha A, Bein T, Fröhlich D, Höfler S, Krenz D, Jauch KW: Simplified acute physiology score (SAPS II) zur Einschätzung der Erkrandkungsschwere bei chirurgischen Intensivpatienten. Chirurg 2002, 73(5):439-442.

58. Metnitz PG, Vesely H, Valentin A, Popow C, Hiesmayr M, Lenz K, Krenn CG, Steltzer $\mathrm{H}$ : Evaluation of an interdisciplinary data set for national intensive care unit assessment. Crit Care Med 1999, 27(8):1486-1491.

59. Schuster HP, Schuster FP, Ritschel P, Wilts S, Bodmann KF: The ability of the simplified acute physiology score (SAPS II) to predict outcome in coronary care patients. Intensive Care Med 1997, 23(10):1056-1061.

60. Osterbrink J, Mayer H, Fiedler C, Ewers A, Haslbeck J, Wirth K, Wordel A, Hannich HJ, McDonough J, Krian A, Laczkovics A, Weyand M, Evers GCM: Inzidenz und Prävalenz postoperativer akuter Verwirrtheit kardiochirurgischer Patienten nach Bypassoperationen sowie Herzklappenersatz. Pflege 2002, 15(4):178-189.

doi:10.1186/1471-2253-14-48

Cite this article as: Fleischer et al:: Does an additional structured information program during the intensive care unit stay reduce anxiety in ICU patients?: a multicenter randomized controlled trial. BMC Anesthesiology 2014 14:48.

\section{Submit your next manuscript to BioMed Central and take full advantage of:}

- Convenient online submission

- Thorough peer review

- No space constraints or color figure charges

- Immediate publication on acceptance

- Inclusion in PubMed, CAS, Scopus and Google Scholar

- Research which is freely available for redistribution 